\title{
Elevated Liver Enzymes in Patients with Type 2 Diabetes Mellitus and Non-alcoholic Fatty Liver Disease
}

Amrendra Mandal ${ }^{1}$, Bikash Bhattarai ${ }^{1}$, Paritosh Kafle ${ }^{1}$, Mazin Khalid ${ }^{1}$, Saikiran K. Jonnadula ${ }^{1}$, Jenny Lamicchane $^{2}$, Rajan Kanth ${ }^{3}$, Vijay Gayam ${ }^{1}$

1. Internal Medicine, Interfaith Medical Center, Brooklyn, USA 2. Internal Medicine, St. John Riverside Hospital, Yonkers, USA 3. Gastroenterology, Carilion Clinic, Roanoke, USA

Corresponding author: Amrendra Mandal, amrendramandal@gmail.com

\section{Abstract}

\section{Background}

Non-alcoholic fatty liver disease (NAFLD) is emerging as the most common chronic liver condition. Approximately 70\% of type 2 diabetes mellitus (T2DM) patients have a fatty liver; the progression to nonalcoholic steatohepatitis (NASH) dramatically increases the risks of cirrhosis and hepatocellular carcinoma. The aim of our study was to assess the profile of liver enzymes in subjects with T2DM and NAFLD.

\section{Method}

This was a cross-sectional clinic-based study in patients with T2DM. An ultrasonography of the abdomen was done in all patients in order to examine the presence of a fatty liver. Body mass index (BMI), lipid profile, and liver enzymes were also analyzed in all patients. Institutional Review Board (IRB) approval was provided by the National Academy of Medical Sciences, Bir Hospital, Nepal. Unpaired $t$-test, Chisquare/Fisher's exact test (for categorical variables), and the Pearson/Spearman correlation test were used to find a significant difference, association, and correlation between two or more groups, respectively. The Statistical Package for Social Sciences (SPSS) ${ }^{\circledR}$ Statistics, version 16 (IBM SPSS Statistics, Armonk, NY) was used to analyse the data.

\section{Results}

The study was carried out in 210 patients, and out of the 210 patients, 119 (56.6\%) were male and 91 (43.3\%) were female. The patients were divided into two groups, i.e., the normal alanine aminotransferase (ALT) group and the elevated ALT group. The mean age of the patients was $56.28 \pm 12.3$ years in the normal alanine aminotransferase (ALT) group and $58.6 \pm 24.7$ in the elevated ALT group. The number of T2DM patients with a fatty liver was 117 (55.7\%) and those with a non-fatty liver was 93 (44.2\%) based on an ultrasonography scan. Subjects with NAFLD had a significantly higher ALT $(\mathrm{p}<0.001)$ but no significant rise in serum aspartate aminotransferase (AST), gamma-glutamyl transferase (GGT), and alkaline phosphatase (ALP) levels. The area under the receiver operating characteristic (AUROC) curve for the prediction of fatty liver

Received 11/05/2018 Review began $11 / 13 / 2018$ Review ended 11/19/2018 Published 11/23/2018

\section{(c) Copyright 2018}

Mandal et al. This is an open access article distributed under the terms of the Creative Commons Attribution License CC-BY 3.0., which permits unrestricted use, distribution, and reproduction in any medium, provided the original author and source are credited. based solely on the ALT was 0.84 with the confidence interval $(\mathrm{CI})$ between 0.76 and $0.92(\mathrm{p}<0.05)$.

\section{Conclusions}

Non-alcoholic fatty liver disease is highly prevalent in patients with T2DM. Timely diagnosis and management of the abnormal liver parameters may help to minimize liver-related morbidity and mortality in the diabetic population.

Categories: Endocrinology/Diabetes/Metabolism, Internal Medicine, Gastroenterology

Keywords: non-alcoholic fatty liver disease (nafld), type 2 diabetes mellitus (t2dm), alanine aminotransferase (alt), alanine transaminase

\section{Introduction}

Nonalcoholic fatty liver disease (NAFLD) is emerging as the most common cause of chronic liver disease in Western countries, as well as worldwide [1]. It is characterized by hepatic steatosis in the absence of excessive alcohol consumption. The spectrum of NAFLD ranges from simple steatosis to non-alcoholic steatohepatitis (NASH), fibrosis, and cirrhosis with its complications, such as decompensation and hepatocellular carcinoma (HCC) [2]. It is expected to become the most important indication for liver transplantation in the near future.

Most patients with NAFLD are asymptomatic and typically are identified when abnormal liver studies are noted on routine laboratory assessment. In particular, the liver enzymes, alanine aminotransferase (ALT), and aspartate aminotransferase (AST) levels are elevated. However, these enzymes may not be elevated in all 
cases of NAFLD, and the level of aminotransferases may not reliably predict the extent of inflammation and cirrhosis [3].

Herein, we describe a study performed to investigate the association between liver enzymes and NAFLD in patients with type 2 diabetes mellitus (T2DM) determined by ultrasonography in a Nepalese population.

\title{
Materials And Methods
}

This was a cross-sectional, observational study conducted between September 2009 and December 2010. Patients with a diagnosis of T2DM who were being treated as outpatients at Bir Hospital were enrolled in the study. The study protocol was approved by the institutional review board (IRB) of the National Academy of Medical Science (NAMS), Bir Hospital. All patients who satisfied the inclusion criteria were recruited in the study. The enrolled patients further underwent a structured health history and physical examination, including measurements of body mass index (BMI). All patients had liver function biomarkers, including alanine aminotransferase (ALT), aspartate aminotransferase (AST), alkaline phosphatase (ALP), gammaglutamyl transferase (GGT), glycosylated hemoglobin (HbA1c), hepatitis B surface antigen (HBsAg), and anti-hepatitis $\mathrm{C}$ antibody studies. The normal ranges for the labs were as follows: ALT: 10 - 40 international units per liter (IU/L), AST: 10 - 35 IU/L, GGT: 5 - 30 IU/L, and ALP: 80 - 290 IU/L. The study subjects with or without NAFLD were divided into groups according to the ALT level.

Patients with alcohol consumption, known liver disease, viral hepatitis, and steatogenic medication were excluded. A detailed history of alcohol consumption was taken with critical level being $<20 \mathrm{~g} /$ day in women and < $30 \mathrm{~g}$ /day in men [4]. Abdominal ultrasound was used as an imaging tool for diagnosing NAFLD [5].

A P value $<0.05$ was set as the level of statistical significance. All analysis was performed by using the Statistical Package for Social Sciences (SPSS) ${ }^{\circledR}$ Statistics, version 16 (IBM SPSS Statistics, Armonk, NY).

\section{Results}

The flow diagram of the study populations with T2DM are shown in Figure 1.

\author{
Study population $(n=429)$ \\ Excluded \\ Alcohol consumption \\ Previously diagnosed hepatitis \\ Acute viral Hepatitis (positive viral serology) \\ Previously diagnosed Liver Cirmosis \\ History of malignancy, Steatogenic drugs \\ Refusal for consent
}

Final study group $(n=210)$

(Male /Female=119:91)

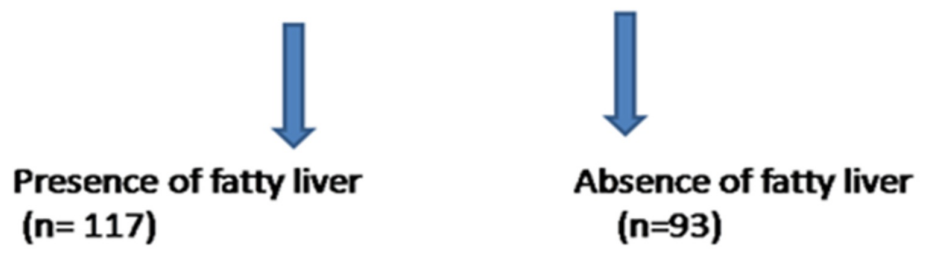

FIGURE 1: Flow Chart of the Study Populations

The baseline characteristics and liver enzymes of patients with T2DM are shown in Table 1. 


\section{Cureus}

\begin{tabular}{|c|c|c|c|}
\hline Characteristics & ALT < 40 IU/L & $A L T \geq 40 \mathrm{IU} / \mathrm{L}$ & P-value \\
\hline Age, years (mean $\pm S D$ ) & $56.28 \pm 12.3$ & $58.6 \pm 24.7$ & NS \\
\hline \multicolumn{4}{|l|}{ Sex } \\
\hline Males n (\%) & $55(26.1)$ & $64(30.4)$ & NS \\
\hline Females n (\%) & $42(20)$ & 49 (23.3) & NS \\
\hline BMI - Kg/m2, (mean) & $28.1 \pm 6.7$ & $30.2 \pm 7.5$ & NS \\
\hline Total cholesterol - mg/dL, (mean) & 208 & 215 & NS \\
\hline HDL - mg/dL (mean) & 42 & 39 & NS \\
\hline Triglyceride - mg/dL (mean) & 187 & 210 & NS \\
\hline Hb A1C (\%) & $6.9 \pm 2.3$ & $7.1 \pm 2.5$ & NS \\
\hline NAFLD n (\%) & $32(27.3)$ & $85(72.6)$ & $<0.001$ \\
\hline
\end{tabular}

\section{TABLE 1: Baseline Characteristics and Liver Enzymes of Patients with Type 2 Diabetes Mellitus}

(T2DM)

BMI: body mass index; Hb A1c: glycosylated hemoglobin; HDL: high-density lipoprotein; n: number; NAFLD: non-alcoholic fatty liver disease; SD: standard deviation

This study was carried out in 210 patients, and of those patients, 119 (56.6\%) were male and 91 (43.3\%) were female. The patients were divided into two groups, i.e., the normal ALT group and the elevated ALT group. The mean age of the patients was $56.28 \pm 12.3$ years in the normal ALT group and $58.6 \pm 24.7$ in the elevated ALT group. The number of T2DM patients with fatty liver was 117 (55.7\%) and those with a non-fatty liver was 93 (44.2\%) based on ultrasonography scans. Subjects with NAFLD had significantly higher ALT levels (p $<0.001$ ) but no significant rise in AST or GGT levels. The relationship of liver biochemistry and enzymes in T2DM with and without fatty liver based on ultrasonography are shown in Table 2.

\begin{tabular}{|c|c|c|c|}
\hline Parameters & DM without fatty liver & DM with fatty liver & p-value \\
\hline ALT & $24.7 \pm 7$ & $51.5 \pm 27$ & $<0.001$ \\
\hline AST & $22 \pm 15$ & $26.1 \pm 13$ & 0.24 \\
\hline GGT & $15 \pm 37$ & $23 \pm 52$ & 0.09 \\
\hline ALP & $132 \pm 56$ & $141 \pm 60$ & 0.08 \\
\hline
\end{tabular}

TABLE 2: Relationship of Liver Biochemistry and Enzymes in T2DM With and Without Fatty Liver Based on Ultrasonography

ALP: alkaline phosphatase; ALT: alanine aminotransferase; AST: aspartate aminotransferase; DM: diabetes mellitus; GGT: gamma-glutamyl transferase; T2DM: type 2 diabetes mellitus

BMI and triglyceride values were higher in fatty liver but were not statistically significant. The duration of T2DM was also significantly associated with elevated liver enzymes, irrespective of the presence of a fatty liver, as shown in Figure 2. 


\section{Cureus}
$\square \mathrm{ALT}<40 \quad \square \mathrm{ALT}>\mathbf{4 0}$
$\mathbf{p}<0.05$

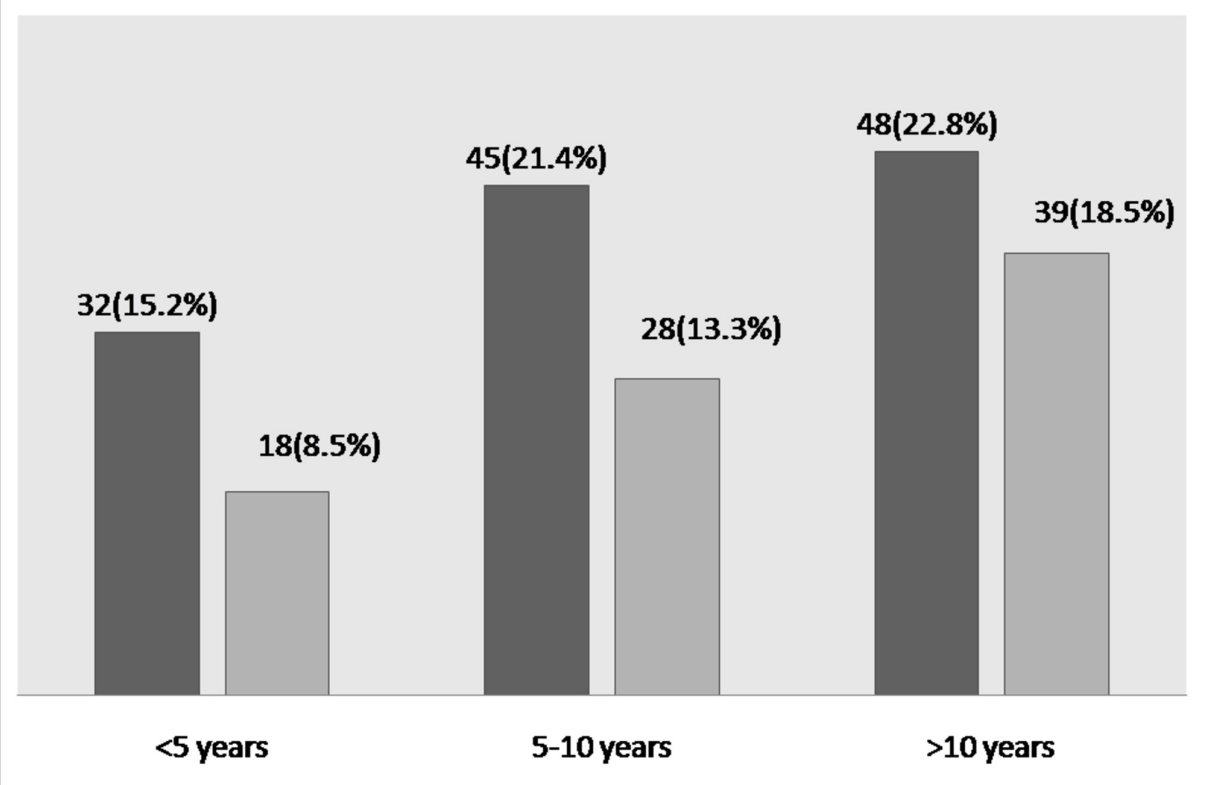

FIGURE 2: Bar Diagram Showing Correlation of ALT with Duration of T2DM Irrespective of the Presence of NAFLD

ALT: alanine aminotransferase; NAFLD: non-alcoholic fatty liver disease; T2DM: type 2 diabetes mellitus

The area under the receiver operating characteristic (AUROC) curve for the prediction of fatty liver based solely on the ALT value was 0.84 with a confidence interval (CI) between 0.76 and $0.92(\mathrm{p}<0.05)$ (Figure 3).

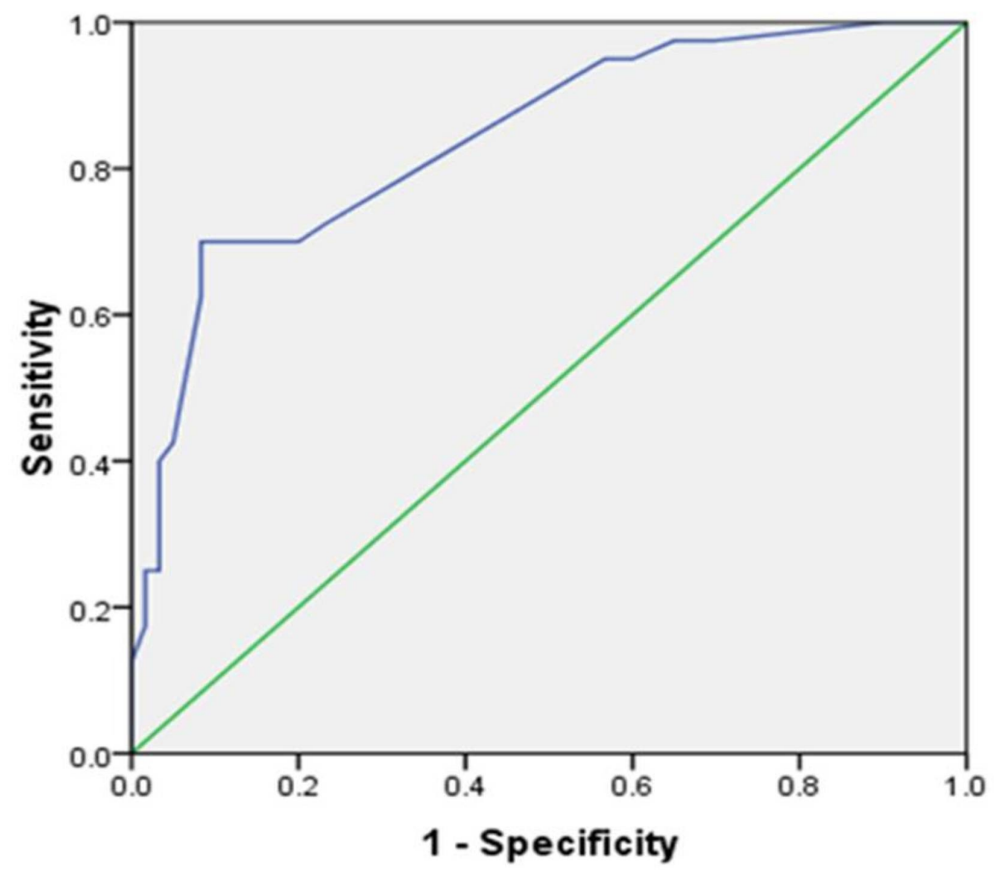

FIGURE 3: Receiver Operating Character (ROC) Curve for ALT in Predicting the Presence of a Fatty Liver

ALT: alanine aminotransferase 


\section{Discussion}

Our results showed that liver enzymes, especially ALT, were independently associated with NAFLD in a Nepalese population with T2DM.

A higher incidence of liver function test abnormalities has been associated with individuals with T2DM than individuals without T2DM [6]. In the present study, the ALT was elevated in $40.4 \%$ of the diabetic population, while the AST and ALP were increased only in $17 \%$ and $16 \%$ of the diabetic population, respectively. Similar to our study, Shrestha et al. and Thanpari et al. from Nepal also observed a statistically significant increase in ALT levels in diabetic patients as compared to the control groups [7-8]. Likewise, a study conducted by $\mathrm{Ni}$ et al. from Malaysia also had a similar pattern with $18 \%, 12 \%$, and $5 \%$ for ALT, AST, and ALP, respectively, in the diabetic population [9]. In the Ghimire et al. study, the values of both transaminases were higher in diabetic females than in male diabetic patients, though not at a statistically significant level [10]. This finding was not observed in our study.

A large United Kingdom study illustrated that NAFLD detected by ultrasonography (USG) was the most common cause of abnormal liver biochemistry [11]. Diabetic subjects with NAFLD had significantly higher ALT, AST, and GGT levels and a significantly lower AST: ALT ratio in comparison with diabetic subjects without NAFLD, but there was no significant difference in the ALP levels. Hence, the controversy relied upon a different increased pattern of liver function tests (LFTs) in several research studies. In addition, a few studies showed AST and some other studies reported GGT as being the most abnormal parameter in their diabetic populations $[7-8,12]$.

Additionally, there are a number of studies that support significantly elevated levels of transaminases in the diabetic population, especially more so with NAFLD than non-NAFLD, including the present study. The ultrasonography results of 210 patients with T2DM in our study showed fatty infiltration in $55.7 \%$ of the patients, which was slightly lower compared to the Prashanta et al., Hernaez et al., and Leite et al. studies with T2DM [13-15]. Higher triglyceride levels were also associated with NAFLD in a study by Leite et al., and among NAFLD patients, they discovered $78 \%$ of the patients to have NASH based on the histopathological study [13]. The presence of high triglycerides, low HDL cholesterol levels, and increased ALT levels were independently associated with a higher risk of histologically confirmed non-alcoholic steatohepatitis (NASH). In another study by Leite et al. in 2011, the presence of NASH was independently associated with high serum GGT levels, older age, and male gender [16]. However, Prashanta et al. confirmed NAFLD by liver biopsy in only $54.11 \%$ of T2DM patients, which was slightly lower than the Leite et al. study [13, 17]. While the simple fatty liver seen in NAFLD does not correlate with increased morbidity or mortality, the progression to NASH dramatically increases the risks of cirrhosis and hepatocellular carcinoma.

ALT is located in the hepatocellular cytosol, whereas AST is located mostly within the mitochondria. In fact, NAFLD and NASH have been reported to be the most common chronic causes of elevated liver enzymes and are often a clue for the need for further diagnostic evaluation [18]. The mechanism of the development of a fatty liver is explained by the insulin resistance that activates lipolysis, resulting in the accumulation of non-esterified fatty acids. This enhanced fat accumulation in the liver is known to be directly toxic to hepatocytes [19]. This results in an increase in ALT [6]. Patients with NAFLD have increased liver-related and overall mortality commonly due to cardiovascular disease [4].

A liver biopsy remains the gold standard for characterizing liver histology in NAFLD, but it is expensive and carries some morbidity and, very rarely, mortality risk. Thus, it should be performed in those who would benefit the most from the diagnostic and prognostic perspectives. Prati et al. have proposed an ALT cut-off of for healthy males and females to be $30 \mathrm{U} / \mathrm{L}$ and $19 \mathrm{U} / \mathrm{L}$, respectively, to be diagnostically useful in NAFLD [20]. Considering the lower cut-off based on the Prati et al. study, the sensitivity for ALT would considerably increase in diagnosing NAFLD.

Based on the ROC curve, our study had an AUROC of 0.84 for ALT in predicting NAFLD in diabetic patients. Routine ultrasound examination of the abdomen (for the presence of NAFLD) may be performed in T2DM patients who have elevated liver enzymes, and subsequently, early treatment and reduction of cirrhosis and its complications may be possible.

\section{Conclusions}

Non-alcoholic fatty liver disease is highly prevalent in patients with T2DM. A timely diagnosis and management of the abnormal liver parameters may help to minimize liver-related morbidity and mortality in the diabetic population.

\section{Additional Information \\ Disclosures}

Human subjects: Consent was obtained by all participants in this study. National Academy of Medical Sciences Institutional Review Board, Bir Hospital, Nepal issued approval 673-2067/68. Animal subjects: All 
authors have confirmed that this study did not involve animal subjects or tissue. Conflicts of interest: In compliance with the ICMJE uniform disclosure form, all authors declare the following: Payment/services info: All authors have declared that no financial support was received from any organization for the submitted work. Financial relationships: All authors have declared that they have no financial relationships at present or within the previous three years with any organizations that might have an interest in the submitted work. Other relationships: All authors have declared that there are no other relationships or activities that could appear to have influenced the submitted work.

\section{References}

1. Williams CD, Stengel J, Asike MI, et al.: Prevalence of nonalcoholic fatty liver disease and nonalcoholic steatohepatitis among a largely middle-aged population utilizing ultrasound and liver biopsy: a prospective study. Gastroenterology. 2011, 140:124-31. 10.1053/j.gastro.2010.09.038

2. Loomba R, Sanyal AJ: The global NAFLD epidemic. Nat Rev Gastroenterol Hepatol. 2013, 10:686-90. 10.1038/nrgastro.2013.171

3. Mofrad P, Contos MJ, Haque M, et al.: Clinical and histologic spectrum of nonalcoholic fatty liver disease associated with normal ALT values. Hepatology. 2003, 37:1286-92. 10.1053/jhep.2003.50229

4. LaBrecque DR, Abbas Z, Anania F, et al.: World Gastroenterology Organisation global guidelines: nonalcoholic fatty liver disease and nonalcoholic steatohepatitis. J Clin Gastroenterol. 2014, 48:467-73.

5. Chalasani N, Younossi Z, Lavine JE, et al.: The diagnosis and management of non-alcoholic fatty liver disease: Practice Guideline by the American Association for the Study of Liver Diseases, American College of Gastroenterology, and the American Gastroenterological Association. Am J Gastroenterol. 2012, 55:200523. $10.1002 /$ hep. 25762

6. Harris EH: Elevated liver function tests in type 2 diabetes . Clin Diabetes. 2005, 23:115-19. 10.2337/diaclin.23.3.115

7. Shrestha N, Bhatt NP, Neopane P, Dahal S, Regmi P, Khanal M, Shrestha R: Hepatic involvement with elevated liver enzymes in Nepalese subjects with type 2 diabetes mellitus. Int J Biochem Res Rev. 2017, 16:18. 10.9734/IJBCRR/2017/31935

8. Thanpari C, Yadav N, Takhelmayum R, Shrewastwa M, Thapa P: Status of antioxidant and liver function in type-2 diabetic patients attending Nepalgunj Medical College. Bali Med J. 2013, 2:1-4.

9. Ni H, Soe HHK, Htet A: Determinants of abnormal liver function tests in diabetes patients in Myanmar . Int J Diabetes Res. 2012, 1:36-41. 10.5923/j.diabetes.20120103.02

10. Ghimire S, Shakya S, Shakya J, Acharya P, Pardhe BD: Abnormal liver parameters among individuals with type 2 diabetes mellitus Nepalese population. Biochem Pharmacol (Los Angel). 2018, 7:243. 10.4172/21670501.1000243

11. Armstrong MJ, Houlihan DD, Bentham L, et al.: Presence and severity of non-alcoholic fatty liver disease in a large prospective primary care cohort. J Hepatol. 2012, 56:234-40. 10.1016/j.jhep.2011.03.020

12. Mathur S, Mehta DK, Kapoor S, Yadav S: Liver function in type-2 diabetes mellitus patients . Int J Sci Stud. 2016, 3:43-47.

13. Leite NC, Salles GF, Araujo AL, Villela-Nogueira CA, Cardoso CR: Prevalence and associated factors of nonalcoholic fatty liver disease in patients with type-2 diabetes mellitus. Liver Int. 2009, 29:113-19. 10.1111/j.1478-3231.2008.01718.x

14. Hernaez R, Lazo M, Bonekamp S, Kamel I, Brancati FL, Guallar E, Clark JM: Diagnostic accuracy and reliability of ultrasonography for the detection of fatty liver: a meta-analysis. Hepatology. 2011, 54:1082-90. 10.1002/hep.24452

15. Targher G, Bertolini L, Padovani R, et al.: Prevalence of nonalcoholic fatty liver disease and its association with cardiovascular disease among type 2 diabetic patients. Diabetes Care. 2007, 30:1212-18. 10.2337/dc062247

16. Leite NC, Villela-Nogueira CA, Pannain VL, Bottino AC, Rezende GF, Cardoso CR, Salles GF: Histopathological stages of nonalcoholic fatty liver disease in type 2 diabetes: prevalences and correlated factors. Liver Int. 2011, 31:700-706. 10.1111/j.1478-3231.2011.02482.x

17. Prashanth M, Ganesh HK, Vimal MV, et al.: Prevalence of nonalcoholic fatty liver disease in patients with type 2 diabetes mellitus. J Assoc Physicians India. 2009, 57:205-10.

18. Vernon G, Baranova A, Younossi ZM: Systematic review: the epidemiology and natural history of nonalcoholic fatty liver disease and non-alcoholic steatohepatitis in adults. Aliment Pharmacol Ther. 2011, 34:274-85. 10.1111/j.1365-2036.2011.04724.x

19. Judi L, Toukan A, Khader Y, Ajlouni K, Khatib MA: Prevalence of elevated hepatic transaminases among Jordanian patients with type 2 diabetes mellitus. Ann Saudi Med. 2010, 30:25-32.

20. Prati D, Taioli E, Zanella A, et al.: Updated definitions of healthy ranges for serum alanine aminotransferase levels. Ann Intern Med. 2002, 137:1-10. 10.7326/0003-4819-137-1-200207020-00006 